\title{
Effectiveness of Neurofeedback Therapy in Children with Separation Anxiety Disorder
}

Seyed Alireza Sadjadi and Peyman Hashemian *

Assistant Professor of Psychiatry, Psychiatry and Behavioral Sciences Research Center, Ibn-e-Sina Hospital, Faculty of Medicine , Mashhad University of Medical Sciences, Mashhad, Iran

*Corresponding author: Peyman Hashemian, Ibn-e-Sina Hospital, Mashhad University of Medical Sciences, Mashhad, Iran, Tel: 0098-915-110-8593; E-mail: hashemianp@mums.ac.ir

Received Date: July 29, 2014, Accepted Date: September 23, 2014, Published Date: September 30, 2014

Copyright: (c) 2014, Peyman Hashemian, et al., This is an open-access article distributed under the terms of the Creative Commons Attribution License, which permits unrestricted use, distribution, and reproduction in any medium, provided the original author and source are credited.

\begin{abstract}
Background: Anxiety disorders are one of the most common psychiatric disorders in children. Its incidence is $2-5 \%$ of children and adolescents under age 18 . Anxiety disorders are more common in girls than boys. It may start in pre-school, but mostly are in age of 7 to 8 years old.

Method: The main objective of this article was to find out the effect of neurofeedback therapy in children with separation-anxiety disorder. Study population was Children from 7 to 12 years old with separation anxiety disorder who were referred to the child psychiatric clinic and they were divided randomly into two groups of 12 . One group $(\mathrm{N}=12)$ received neurofeedback therapy and the other group $(\mathrm{N}=12)$ received sham neurofeedback therapy (placebo). Data was analyzed with t- test by 21 th version SPSS software.
\end{abstract}

Results: According to calculated t-test in neurofeedback group (8.18), neurofeedback was effective in reducing separation anxiety and the efficacy of treatment was great. But according to calculated t-test in sham group (4.42), reduction of separation anxiety was moderate. Therefore the efficacy of treatment in neurofeedback and sham groups was different.

Conclusion: The results revealed that the efficacy of treatment of neurofeedback and sham groups on separation anxiety in children is different. Comparison of efficacy shows that effectiveness of neurofeedback treatment on separation anxiety was much more in the group treated with Neurofeedback than in the sham group.

Keywords Separation anxiety disorder; Neurofeedback; $\alpha$ wave; $\sigma$ waves

\section{Introduction}

Anxiety disorders are one of the most common psychiatric disorders in children. Incidence is $2-5 \%$ of children and adolescents under age 18. It is more common in girls than boys. It may start in pre-school, but most patients are in age of 7 to 8 years. The importance and necessity of the design is the efficacy of cognitivebehavior therapy where drugs are partially effective; so it is important to find out a way to treat the disorder.

In literature the effect of neurofeedback therapy for other anxiety disorders has been studied but not for separation anxiety disorder of children. The aim of this research was to evaluate the effect of neurofeedback in treatment of children with separation anxiety disorder. In neurofeedback therapy, person can manage his/her own brain waves. For high concentration, one must reinforce Beta waves; for High relaxation, theta waves; and for relaxed awareness, Alpha waves. Each person has a special pattern of brain activity that is associated with specific psychiatric abnormalities. For example in hyperactivity, it is necessary to decrease alpha and theta and enhance beta waves. Brain signals are in the form of Visual or auditory.
Favorable changes in brain waves are followed by audio or visual reward signal. Visual signals can be in forms of graph, number, waves, color or pattern changes, or animation.

Hardt and Kamiya in 1978 could recover anxiety by increasing alpha waves for at least 5 hours training [1]. Taylor and colleagues showed that some symptoms of anxiety are indicative of risk for later onsets of manifestations of these disorders [2]. Corydon Hammond reviewed the effects of the use of neurofeedback in patients with anxiety disorders [3]. In his review, positive findings support the treatment of anxiety disorders. In his literature review article, Moore (2000) also showed 7 from 8 studies had good results [3]. Garrett and Silver in 1976 showed that increased a wave caused decreased anxiety [4]. In 2001 Hammond achieved beneficial effect in the treatment of 21 year old patient with chronic fatigue syndrome using combination of neurofeedback and self-hypnosis [5]. Hammond investigated the effectiveness of neurofeedback in the treatment of OCD [6] and in 2004 evaluated the effects of neurofeedback on different types of OCD [7]. Vanathy, Sharma, and Kumar found that in subjects with general anxiety disorder, theta neurofeedback training is as effective as alpha neurofeedback training, which would result in reducing anxiety and therefore increasing life quality [8]. 


\section{Method}

The purpose of this study was to investigate the effect of real neurofeedback treatment versus sham neurofeedback treatment (placebo) in patients with separation anxiety disorder without any drug therapy and to find out which one is more effective. This research is based on pre-test and post-test. Patients were selected from those attended to child and adolescent psychiatric clinics of Mashhad Medical University, who were diagnosed with separation anxiety disorder that confirmed by two child and adolescent psychiatrists using criteria of DSM IV.

\section{Further selecting criteria were}

Subjects should have had no other psychiatric or neurological disorders in psychiatric interview. Wechsler intelligence score for children (IQ score) should have been above 70 that have been conducted by an expert psychologist.

Informed consent form must have been completed by parents.

In this study, 24 children, aged between 7 and 12, who according to psychiatric interview (based on DSM IV criteria) were diagnosed with separation anxiety disorder were finally selected. Then the selected patients were randomly assigned to control and experimental groups. Each group contained 6 girls and 6 boys. Spencer Anxiety questionnaire was answered by all children' parents. One group $(\mathrm{N}=12)$ received neurofeedback treatment and the other group $(\mathrm{N}=12)$ received non-real neurofeedback treatment (sham). Before the first session and immediately after 20 sessions of neurofeedback therapy, Spencer anxiety test for all subjects were recorded. Neurofeedback treatment was performed as unipolar on the basis of PROCOMP 2 program. At first session, threshold level of each patient was defined. In every session, EEG signals were obtained and the ratio of alpha / theta in $\mathrm{F} 3$ region was measured and a reward ( $500 \mathrm{~Hz}$ tone) is given between 60 to 80 percent of times. The patient is trained to enhance ratio of alpha / theta in F3 throughout the twenty sessions. Threshold level was assigned every three minutes. In subsequent treatment session, at first, threshold was calculated as the mean threshold of the previous session. While constantly recording EEG from the chosen lead, alpha/ theta ratio was calculated every $20 \mathrm{~ms}$ (millisecond), with an interval of $5 \mathrm{~ms}$ and was compared to threshold level. If the ratio was higher than the threshold, reward was given.

At the start of each session, chocolate was promised to each child for a good practice. Each child received 20 sessions and each session was about 30 minutes long. Treatment period lasted 10-12 weeks. During treatment period, ratio of alpha/theta at the beginning and end of each session was recorded. In sham group, all conditions were exactly the same as of real neurofeedback, except that someone else's recorded neurofeedback film was played to them. At the first session, baseline was set and mean of alpha / theta ratio was obtained for each subject. Before the first and at the end of the twentieth session, Spencer test was conducted. Obtained data has been analyzed by SPSS software by using T-test.

\section{Results}

The study sample consisted of 24 students, who were randomly assigned into two groups of real neurofeedback and sham. The two groups were treated equally. Each group consisted of 6 boys and 6 girls. Significance test for comparison of means of age (t-independent) shows the age difference between the two groups is not significant
$(\mathrm{P}=0.1)$. Also, the mean educational level of the two groups (in terms of school year) shows one year difference. However, t-independent test showed no significant difference between the mean educational level of the two groups $(t=2.02 \& \mathrm{P}=0.055)$. As can be seen in Table 2 , in the pre-test anxiety scores, there is a 4 score difference in the means of the two groups. However, after the intervention, the mean anxiety score was reduced (improved) about 37 in real neurofeedback group, but 29 in sham group. Comparison of the two groups' score at baseline (pretest): For homogeneity of the two groups, independent t-test was used. Also homogeneity of variances was measured using Levin F test.

According to the results above, independent $t$ test is not significant, therefore due to anxiety mean score, both groups at baseline (pre-test) are the same and on the basis of F Levine test which is not significant, homogeneity of variance between two groups have also been met (Table1)

According to the above results, calculated t-test (8.18) is significant. Therefore, treatment is also effective in children with separation anxiety. Cohen's $\mathrm{d}$ as a measure for effect size was assessed and showed that the effect size of Neurofeedback in treatment of separation anxiety in children was high (Table 2)

According to the above results, calculated t-test (4.42) is significant. Therefore, the sham treatment group is also effective in reducing children's separation anxiety, and D Cohen as an effect size index shows the effectiveness of the sham treatment group was medium.To compare the efficacy of treatment of real neurofeedback and sham groups on separation anxiety in children, the mean differences between two groups were compared by using independent t-tests. (Table 3) According to the above results, the calculated $t$ index (2.66) is significant. Thus, the efficacy of treatment of real neurofeedback and sham groups on separation anxiety in children are different. Comparison shows the effectiveness of neurofeedback on children with separation anxiety is more than the sham group.

\section{Discussion}

Neurofeedback is a process to record the feedback of biological brain waves in psychophysiological area which has been used since 1960. Research evidences in emotion indicates that left frontal area is associated with more positive affect and memories, whereas the right hemisphere is more involved in negative emotion. A biologic predisposition to depression exists when there is a frontal asymmetry in brain wave activity, with more left frontal alpha activity. This imbalance with more left frontal alpha means that the left frontal area is less activated. Anxiety often involves excess right frontal beta wave activity. Someone who obsessively worries may have this beta excess along the midline or in the center of the top of the head at an electrode location known as $\mathrm{Cz}$. Many studies support these findings.

This study shows that neurofeedback treatment is highly effective in reducing separation anxiety and in sham treatment it had medium effect in reducing separation anxiety. Therefore real neurofeedback therapy on separation anxiety in children was more effective than sham group. It seems that neurofeedback effect on separation anxiety disorder is as effective as on other anxiety disorders such as generalized anxiety disorder, and obsession. Below are some examples:

Moore NC showed that Alpha, theta and alpha-theta enhancements are effective in treatment of anxiety disorders [9]. Rice and colleague showed alpha-increase neurofeedback caused significant reductions in heart rate and decreased self-report of anxiety at 6 weeks 
Citation: Hashemian P, Sadjadi SA (2014) Effectiveness of Neurofeedback Therapy in Children with Separation Anxiety Disorder. J Psychiatry

Page 3 of 3

post treatment [10]. Watson showed an EEG alpha neurofeedback treatment in the management of anxiety [11]. Wiedemann Showed frontal brain waves' asymmetry as a biological substrate of emotions in patients with panic disorders [12]. Biriukova Showed clinical and neurophysiological efficacy of neurofeedback in the combined therapy of anxiety disorders resistant to psychopharmaco therapy [13]. La Vaque and colleagues in evaluating neurofeedback treatment of anxiety disorders showed it was an efficacious treatment [14].

\section{Limitations}

Due to the lack of similar studies on separation anxiety disorder, comparison was not possible.

\section{Suggestions}

Other research on the effectiveness of neurofeedback in children with separation anxiety must be done with placing electrodes on the other regions on the scalp.

\section{Acknowledgement}

I would like to thank Dr. Paria Hebrani for confirming the diagnosis of selected patients as the second child and adolescent psychiatrist.

\section{Implications for Educators}

Psychiatrists can consider neurofeedback as one way of treating separation anxiety disorder.

Neurofeedback therapist can use alpha enhancement to reduce anxiety.

Neurofeedback therapist can use F3 region for treatment of separation anxiety disorders.

\section{References}

1. Hardt JV, Kamiya J (1978) Anxiety change through electroencephalographic alpha feedback seen only in high anxiety subjects. Science 201: 79-81.
2. Wolitzky-Taylor K1, Dour H, Zinbarg R, Mineka S, Vrshek-Schallhorn S, et al. (2014) Experiencing core symptoms of anxiety and unipolar mood disorders in late adolescence predicts disorder onset in early adulthood. Depress Anxiety 31: 207-213.

3. Hammond DC (2005) Neurofeedback with anxiety and affective disorders. Child Adolesc Psychiatr Clin N Am 14: 105-123, vii.

4. Hammond DC (2005) Neurofeedback Treatment of Depression and Anxiety. Journal of Adult Development 12: 131-137.

5. Hammond DC (2001) Treatment of chronic fatigue with neurofeedback and self-hypnosis. NeuroRehabilitation 16: 295-300.

6. Hammond DC (2003) QEEG-guided neurofeedback in the treatment of obsessive compulsive disorder. Journal of Neurotherapy 7: 25-52.

7. Hammond DC (2004) Treatment of the obsessional subtype of obsessive compulsive disorder with neurofeedback. Biofeedback 32: 9-12.

8. Budzynski HK, Evans JR, Abarbanel A (2009) Introduction to Quantitative EEG and Neurofeedback: Advanced Theory and Applications. Academic Press: 304-311.

9. Vanathy S, Sharma PSVN, Kumar KB (1998) the efficacy of alpha and theta neurofeedback training in treatment of generalized anxiety disorder. Indian Journal of Clinical Psychology 25: 136-43.

10. Moore NC (2000) A review of EEG biofeedback treatment of anxiety disorders. Clin Electroencephalogr 31: 1-6.

11. Rice KM1, Blanchard EB, Purcell M (1993) Biofeedback treatments of generalized anxiety disorder: preliminary results. Biofeedback Self Regul 18: 93-105.

12. Watson BW, Woolley-Hart A, Timmons BH (1979) Biofeedback instruments for the management of anxiety and for relaxation training. J Biomed Eng 1: 58-62.

13. Wiedemann G1, Pauli P, Dengler W, Lutzenberger W, Birbaumer N, et al. (1999) Frontal brain asymmetry as a biological substrate of emotions in patients with panic disorders. Arch Gen Psychiatry 56: 78-84.

14. Biriukova EV, Timofeev IV, Mosolov SN (2005) Clinical and Neurophysiological Efficacy of Neurofeedback in the Combined Therapy of Anxiety Disorders Resistant to Psychopharmacotherapy. In: European Neuropsychopharmacology, Elsevier 15: 162-163. 